\title{
The Role of Spiking Local Interneurons in Shaping the Receptive Fields of Intersegmental Interneurons in the Locust
}

\author{
Gilles Laurent \\ Department of Zoology, University of Cambridge, Cambridge CB2 3EJ, UK
}

Intracellular recordings were made simultaneously from pairs of spiking local and intersegmental interneurons in the mesothoracic ganglion of the locust Schistocerca gregaria (Forskal). The spiking local interneurons belong to a midline group, and the intersegmental interneurons to a lateral-posterior population that projects ipsilaterally to the metathoracic ganglion. Both respond to mechanosensory inputs from one middle leg. The receptive fields of both types of interneurons were plotted and the contribution of the local interneurons to the shaping of the receptive fields of the intersegmental interneurons was studied.

The spiking local interneurons are excited by specific arrays of exteroreceptors and proprioceptors of one leg. They make inhibitory connections with specific intersegmental interneurons which thus have corresponding inhibitory regions to their receptive fields. For example, inhibition from local interneurons with inputs from hairs or campaniform sensilla on the tarsus could sharpen the boundaries of the excitatory receptive field of an intersegmental interneuron by lateral inhibition. Inhibition from local interneurons with inputs from joint proprioceptors suppresses the excitation of an intersegmental interneuron during the antipreferred direction of movement. Rapid alternate movements about one joint can in this way be signaled by an intersegmental interneuron in a phase-locked manner. Certain intersegmental interneurons are excited by exteroreceptive inputs and inhibited by proprioceptive signals, or vice versa. The inhibition could become significant during walking, where the excitatory and inhibitory inputs to an interneuron are likely to be evoked during the different phases of a step cycle.

The inhibitory regions of the receptive field of an intersegmental interneuron have never been found to be smaller than the excitatory regions of the receptive field of a presynaptic local interneuron and can result from converging inhibitory inputs from several spiking local interneurons. The inhibitory connections are highly specific: Several local interneurons may be excited by a particular stimulus that also inhibits an intersegmental interneuron, but only a small proportion of the former are the cause of the inhibition in the intersegmental interneuron.

\footnotetext{
Received Feb. 6, 1987; revised Mar. 30, 1987; accepted Apr. 1, 1987.

This work was supported by an SERC (UK) grant to M. Burrows. I am graleful to Malcolm Burrows, Reinhold Hustert, Debby Kuterbach, Kay Seymour, and Alan Watson for their helpful comments on the manuscript.

Correspondence should be addressed to G. Laurent, Department of Zoology, University of Cambridge, Downing Street, Cambridge CB2 3EJ, England.

Copyright (C) 1987 Society for Neuroscience $0270-6474 / 87 / 092977-13 \$ 02.00 / 0$
}

To build a central representation of the world, the nervous system first extracts the available information through many sensory channels that each encode in parallel a fraction of this information. The sensory inputs are then combined centrally to acquire a given significance and to lead to a sensation, the perception of a sensory experience and/or a motor act.

In mammals, central encoding of somatosensory signals first occurs in the dorsal gray matter of the spinal cord with convergence of inputs of different modalities and origins onto single targets. The sensation of thermoreceptive, nociceptive and mechanoreceptive afferences thus starts in the dorsal horn with the activation of second-order neurons that have complex receptive fields (Price, 1986). Some of these neurons also seem to be involved in mediating local reflexes (Cervero, 1986). The number of neurons implicated, however, is so enormous that, at present, it is difficult to establish the patterns of connectivity that underlie this already complex sensory integration.

In insects, most mechanosensory signals from leg proprioand exteroreceptors are fed directly to local interneurons and some in parallel to motor neurons. The pathways that underlie local reflexes elicited either by proprio- or exteroreceptors can now be described in great detail in terms of connections between identified neurons (Wong and Pearson, 1976; Burrows and Siegler, 1982; Siegler and Burrows, 1983, 1986; Burrows, 1987a, b). The proprioceptive and exteroreceptive signals are also transmitted in parallel to intersegmental interneurons, so that one leg is informed of the actions of its neighbor (Laurent, 1986, $1987 \mathrm{a})$. The excitatory regions of the receptive fields of these intersegmental interneurons are determined by the arrays of receptors of the leg from which they receive their inputs (Laurent, $1987 \mathrm{~b}, \mathrm{c})$. A large proportion of these intersegmental interneurons also has inhibitory regions to their receptive fields. Since no mechanosensory neuron is known to make inhibitory connections in the central nervous system of insects, at least one layer of interneurons must be interposed between the afferents and the intersegmental interneurons. Spiking local interneurons in a population that mediates reflex inhibition of motor neurons (Burrows and Siegler, 1982) and of nonspiking local interneurons (Burrows, 1987b) are the intercalated interneurons (Laurent, 1987c).

The aim of this paper is therefore to examine the role played by these spiking local interneurons in the elaboration of the inhibitory receptive fields of the intersegmental interneurons. It is shown that the local interneurons, by virtue of specific monosynaptic inhibitory connections with the intersegmental interneurons, play a key role in shaping their sometimes complex response characteristics. The function of the coexistenceand sometimes juxtaposition - of excitatory and inhibitory re- 

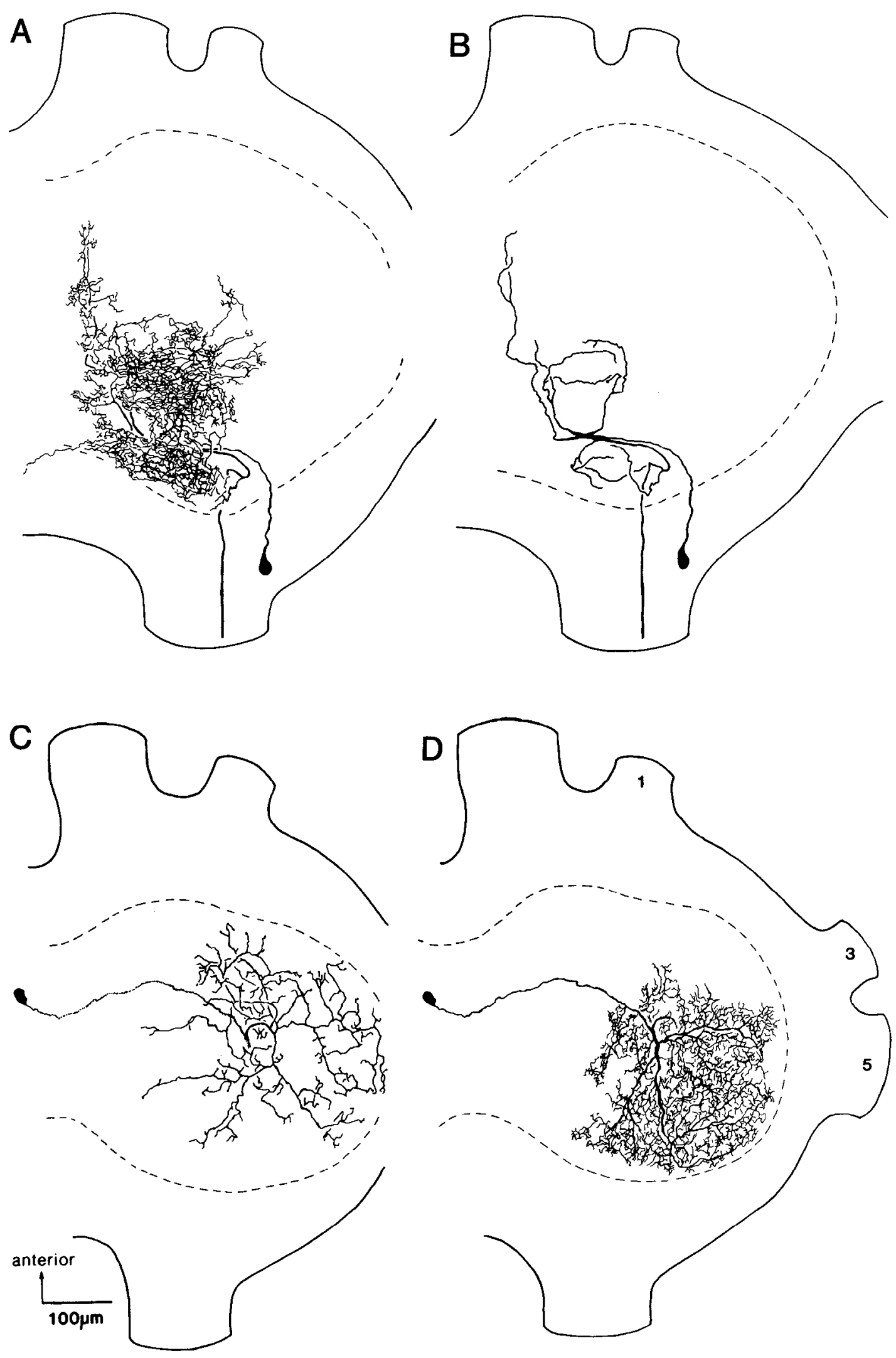

Figure 1. Morphology of mesothoracic intersegmental and spiking local interneurons studied in this paper, as seen in whole-mount. $A$, $B$. An intersegmental interneuron, inhibited by the extension of the ipsilateral tibia. Its fine branches $(A)$ in the dorsal neuropil arise from the primary and secondary neurites $(B)$. The axon runs in the ipsilateral connective to the metathoracic ganglion. $C, D$, A spiking local interneuron of the midline group that is excited by exteroreceptors on the ventral surface of the tibia. Dorsal $(C)$ and ventral $(D)$ fields of branches. In dorsal views, dashed lines indicate edges of neuropil. 


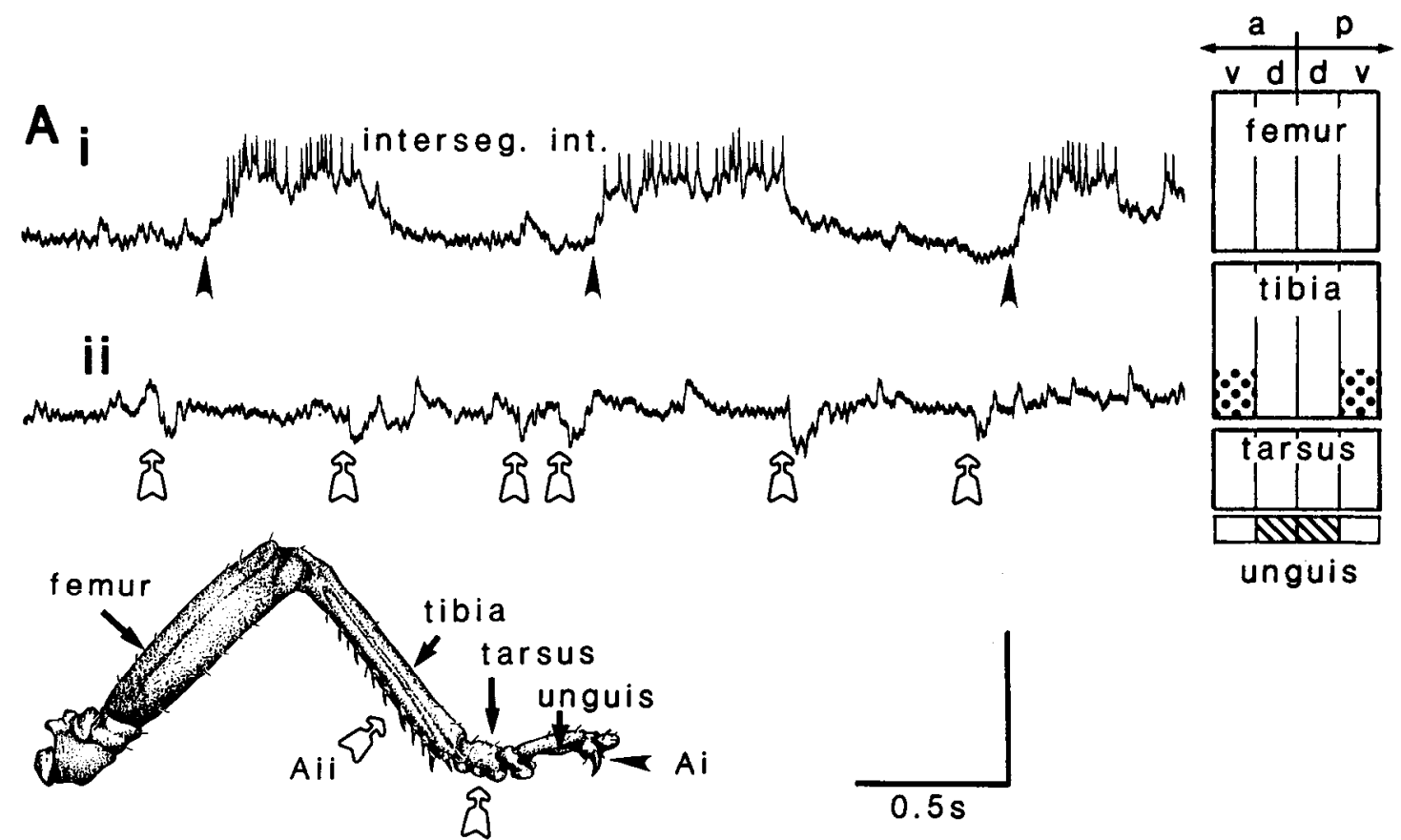

B

B

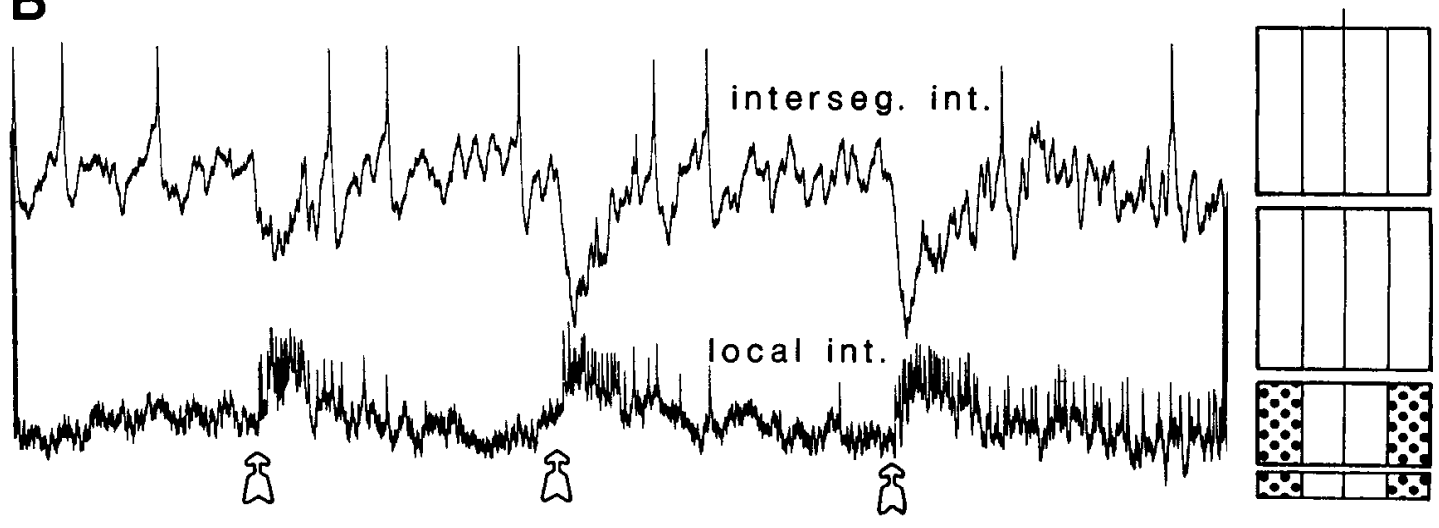

Figure 2. Responses of 2 intersegmental interneurons to exteroreceptive stimuli. $A_{i}$, An interneuron that is depolarized and spikes when hairs on the unguis (see inset drawing of middle leg) are touched (filled arrowheads). $A_{i i}$, If the ventral surface of the tibia is touched (open arrows), the interneuron is hyperpolarized by a barrage of IPSPs. Right panel, Diagrammatic receptive field of this interneuron. Hatched area, excitatory; stippled area, inhibitory (see also Fig. 11). B. Another intersegmental interneuron that normally spikes tonically is strongly inhibited by a barrage of IPSPs when the ventral surface of the tarsus is touched (open arrows). A spiking local interneuron recorded simultaneously (bottom trace) is, by contrast, strongly excited by this stimulus. Right panel, receptive field of the intersegmental interneuron. $a$, Anterior; $d$, dorsal; int., interneuron; interseg., intersegmental; $p$, posterior; $v$, ventral. Calibrations: Vertical, $5 \mathrm{mV}(A)$; intersegmental, $5 \mathrm{mV}$, local, $2.5 \mathrm{mV}(B)$.

gions in the receptive fields of the intersegmental interneurons is considered in the context of locomotor behavior.

\section{Materials and Methods}

Adult locusts (Schistocerca gregaria; Forskal) of either sex were taken from our crowded laboratory culture. They were mounted in plasticine, ventral side up, with all 6 tibiae and tarsi free to move. The meso- and metathoracic ganglia were exposed and fixed on a wax-coated platform. The thoracic cavity was perfused with fresh saline throughout the exneriments. The sheath of the mesothoracic ganglion was treated for 12 min with a $1 \%(\mathrm{wt} / \mathrm{vol})$ solution of protease (Sigma type XIV) in saline.

Extereroceptive stimulation of a mesothoracic leg was carried out by hand, using a fine paintbrush to move hairs or the tip of a needle to press on campaniform sensilla. Proprioceptive stimulation resulting from imposed movements of the tibia or the tarsus was applied either by hand or by using a servomotor driven by a function generator.

In some experiments, muscular activity was recorded extracellularly, using pairs of insulated $50 \mu \mathrm{m}$ stainless steel wires inserted through the femoral cuticle. Sensory spikes were recorded from the tarsal nerves in the tibia (N5B2a and b) after immobilization of the latter, using extracellular hook electrodes isolated from the saline with silicon or paraffin oil.

Intracellular recordings were made from the somata of at least 30 different intersegmental interneurons in an identified mesothoracic population (Laurent, 1987a), of spiking local interneurons in a midline group (Burrows and Watkins, 1986) and of motor neurons. These precise recording sites, coupled with careful definition of the response properties, lead to reliable identification of the interneurons. Further confirmation of identity was provided by intracellular staining. Glass micropipettes were filled with $2 \mathrm{M}$ potassium acetate or a $6 \%$ solution of hexammine cobaltic chloride (Brogan and Pitman, 1981) for later staining. In 81 paired recordings from a local and an intersegmental interneuron, $6(7.5 \%)$ inhibitory interactions were found. The records were stored on a 7-channel Racal FM tape recorder for later averaging, or for display on a Gould ES1000 printer or a Nicolet digital oscilloscope and Philips XY plotter. 
Figure 3. A spiking local interneuron is responsible for the inhibition of an intersegmental interneuron. $A$, This intersegmental interneuron is excited by touching hairs on the dorsal tarsus (filled arrowheads). If the hairs on the ventral tarsus are touched (open arrows), the spiking local interneuron spikes faster and the intersegmental interneuron is hyperpolarized. The bursts of sensory spikes in the tibial nerve $5 \mathrm{~B} 2 \mathrm{~b}$ are from pad afferents on the ventroposterior tarsus. $B$, Receptive field of the intersegmental interneuron. $C$, Injecting current into the local interneuron to make it spike faster evokes IPSPs in the intersegmental interneuron that follow $1: 1 . D$, Signal average of 512 sweeps triggered from the spikes of the local interneuron. The IPSP follows with a latency of about $1 \mathrm{msec}$. Calibration: Horizontal, $0.5 \mathrm{sec}(A, C) ; 30 \mathrm{msec}(D)$; vertical, interseg., $2.5 \mathrm{mV}(A, C)$; local, $5 \mathrm{mV}$. Abbreviations and symbols as in previous figures.

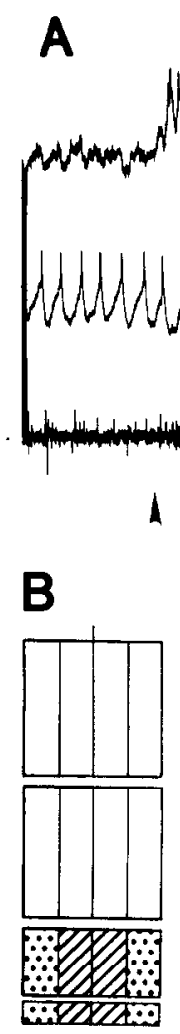

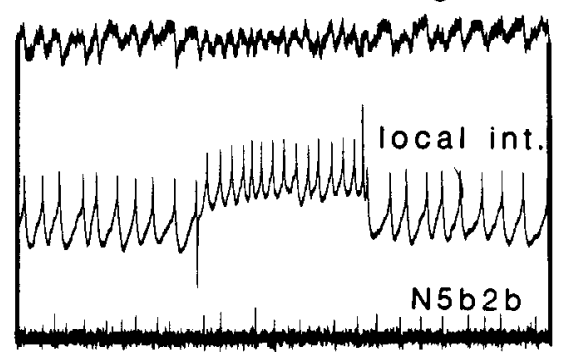

interseg. int.

interseg. int.

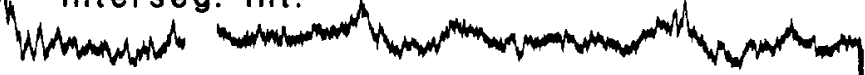

local int.

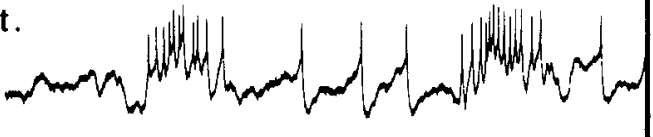

N5b2b

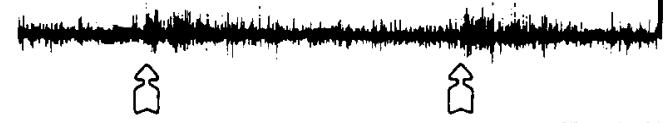

\}

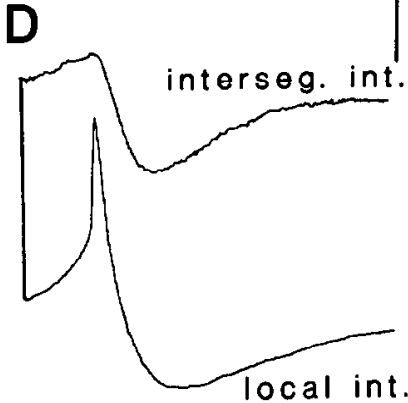

A

B

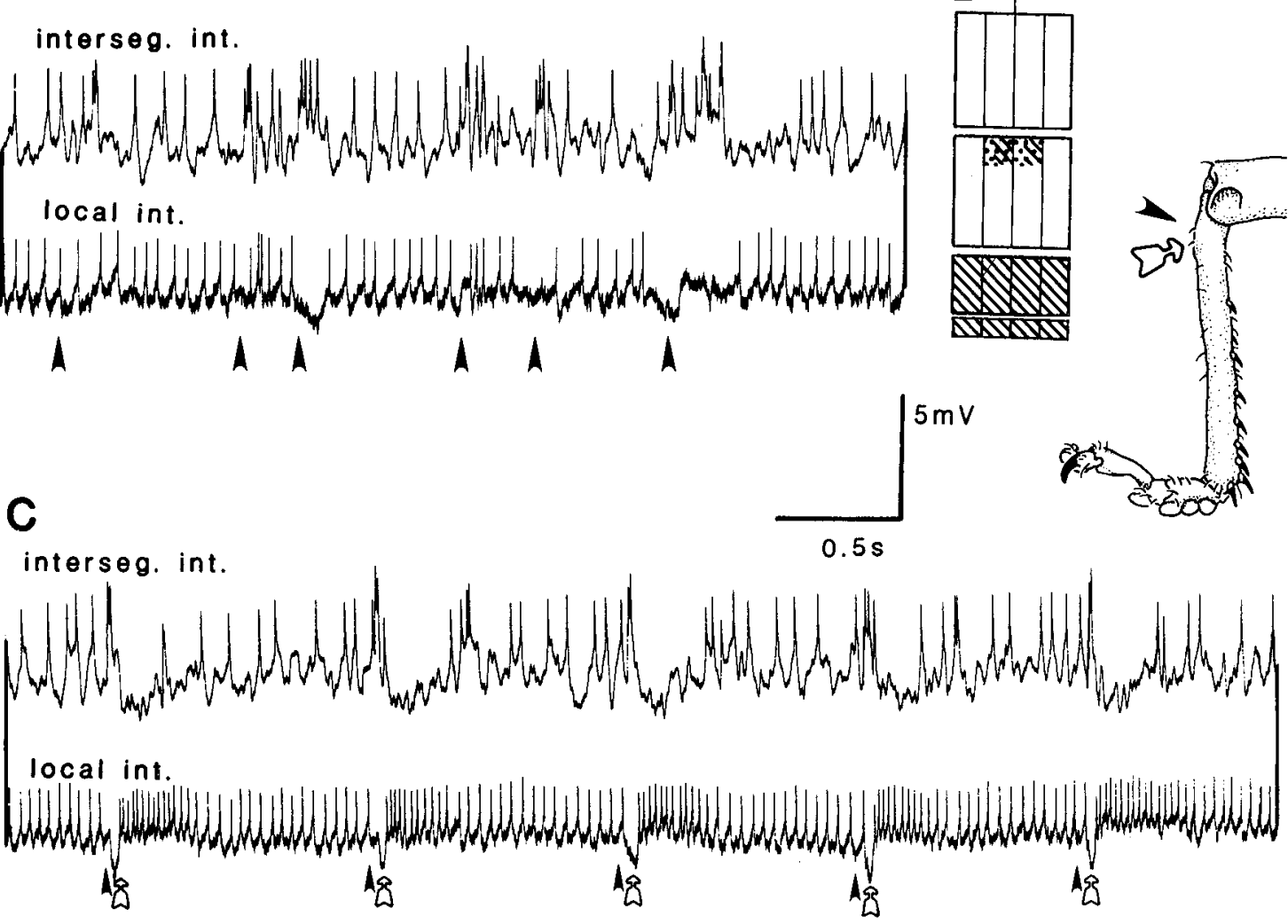

Figure 4. An intersegmental interneuron with inhibitory and excitatory inputs from the same region of the leg. $A$, Touching hairs on the knee joint (inset) excites the intersegmental interneuron (filled arrowheads) and slightly inhibits a local interneuron. $B$, Receptive field of the intersegmental interneuron. $C$, Pressing on the knee joint (open arrows) at the same location as in $(A)$ makes the local interneuron spike faster and inhibits the intersegmental interneuron. Just before (and sometimes after) each stimulus is applied, hairs on the knee are inevitably touched, as they cover the area where the cuticular receptors are located (arrowheads). As a result, the intersegmental and local interneurons are, respectively, phasically excited and inhibited. 

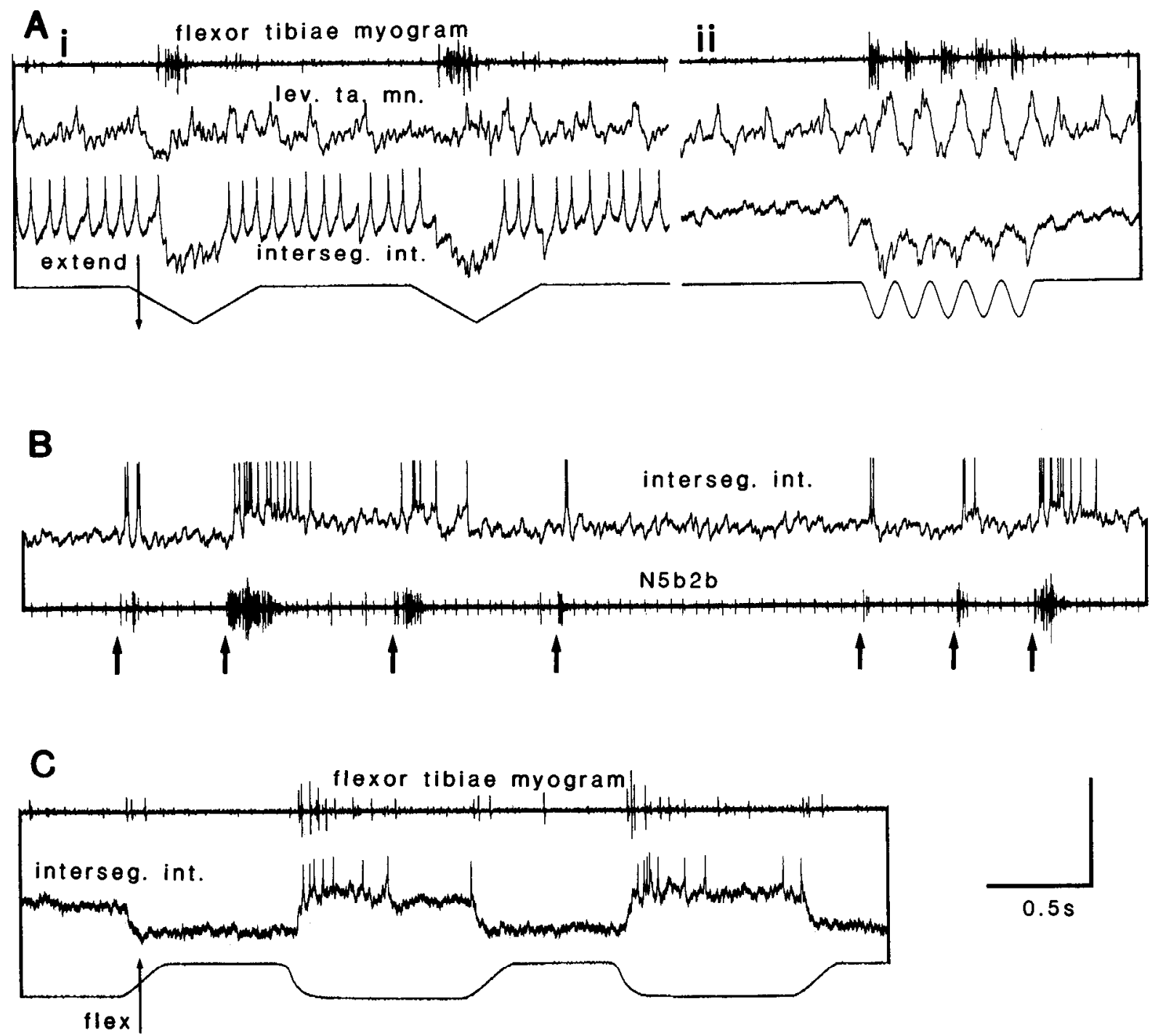

Figure 5. Responses of intersegmental interneurons to proprioceptive stimuli. $A$, An interneuron is inhibited by a barrage of IPSPs during an imposed extension of the tibia. $A_{i}$, The interneuron is at normal membrane potential. $A_{i}$, The interneuron has been hyperpolarized below spike threshold; the flexion phase of the imposed movement does not evoke EPSPs in the interneuron. The levator tarsi motor neuron (lev. ta. mn.) is also affected by this movement in an interjoint local reflex. By contrast to the interneuron, its membrane potential follows the waveform. $B$. The same interneuron is excited only by intrinsic movement of the tarsus, as monitored by the sensory activity in tibial nerve $5 \mathrm{~b} 2 \mathrm{~b}$. Arrows indicate the onset of imposed movement. $C$, A different interneuron is hyperpolarized by flexion and depolarized by extension of the tibia. Calibration: Vertical: interneuron, $12.5 \mathrm{mV}(A)$; motor neuron, $12.5 \mathrm{mV}$, interneuron, $5 \mathrm{mV}(B)$; interneuron, $6.25 \mathrm{mV}(C)$.

For intracellular staining, cobalt was iontophoretically injected using $0.5 \mathrm{sec}$ square pulses of positive current $(5-10 \mathrm{nA})$ at $1 \mathrm{~Hz}$ for $10 \mathrm{~min}$. The neurons were revealed in ammonium sulfide, subsequently intensified with silver (Pitman et al., 1972; Bacon and Altman, 1977), and drawn as whole-mounts with a tube attached to a compound microscope.

\section{Results}

The intersegmental interneurons have their somata clustered at the lateral base of the posterior connectives (Fig. 1, $A, B$ ), and arborize on the side of the ganglion ipsilateral to the middle leg from which they receive inputs (Laurent, 1986, 1987a). The spiking local interneurons have their somata clustered at the ventral midline (Fig. 1, $C, D$ ) and have 2 fields of branches, again ipsilateral to the leg supplying their inputs; ventrally, are fine branches (Fig. 1D) on which most input synapses are found, and dorsally are varicose branches (Fig. 1C) from which mainly output synapses are formed (Watson and Burrows, 1985; Burrows and Watkins, 1986).

\section{Processing of exteroreceptive information}

Among this population of intersegmental interneurons are some that respond with great specificity to tactile stimulation of one middle leg. The receptive field of such an interneuron is often composed of excitatory and inhibitory regions, which are not necessarily contiguous. One, for example, is excited and spikes for as long as hairs on the unguis are moved (Fig. $2 A, i$ ). If, however, hairs on the ventral and distal region of the tibia are touched, the interneuron is hyperpolarized by a barrage of IPSPs (Fig. 2A, $i i$ ).

Other interneurons are affected by just one region of the leg, and that region can provide both excitatory and inhibitory inputs. The interneuron in Figure $2 B$, for example, is strongly 

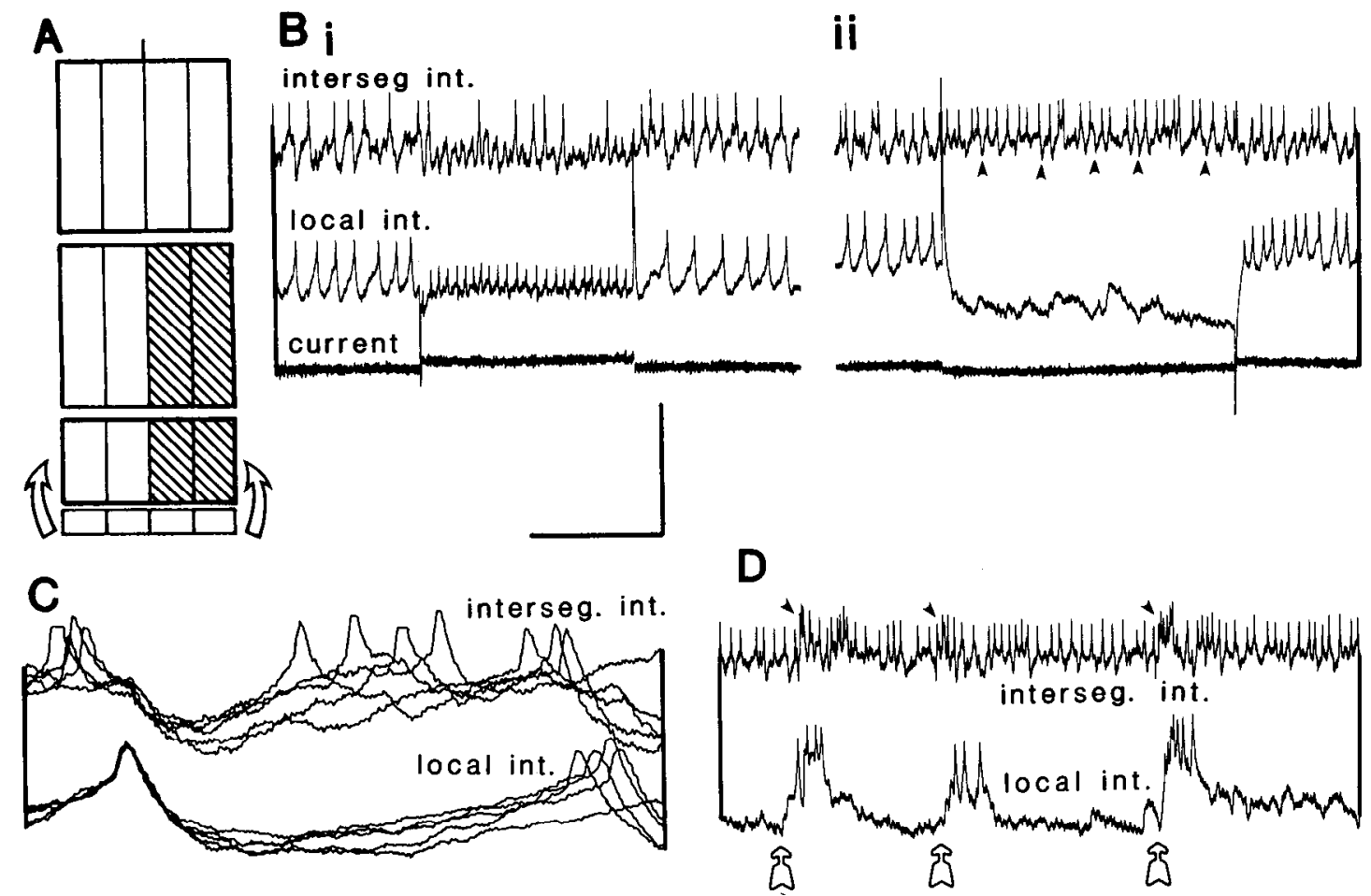

Figure 6. The mixing of mechanosensory modalities. An intersegmental interneuron is excited by exteroreceptive inputs and inhibited by proprioceptive ones. $A$, Receptive field of the intersegmental interneuron. It is excited by touching the posterior tibia and tarsus, and inhibited by a retraction of the unguis. $B_{i}$, Injecting depolarizing current into a local interneuron excited by the retraction of the unguis to make it spike faster inhibits the intersegmental interneuron. $B_{i b}$, Injecting hyperpolarizing current into the local interneuron to suppress its tonic spikes disinhibits the intersegmental interneuron. Some IPSPs in the intersegmental interneuron still remain (arrowheads). $C$, Superimposed sweeps triggered from spikes in the local interneuron. The latency to the IPSPs is constant at about $1 \mathrm{msec} . D$, If the tarsus is touched, the intersegmental interneuron is excited (arrowheads). If the unguis is moved at the same time, the local interneuron is made to spike (open arrows), and this reduces the spike frequency in the intersegmental interneuron. Calibration: Horizontal, $0.5 \mathrm{sec}(B, D) ; 16.5 \mathrm{msec}(C)$; vertical, $5 \mathrm{mV}(B, D) ; 3 \mathrm{mV}(C)$.

hyperpolarized only when the ventral pads of the tarsus are touched. The pattern of IPSPs at each stimulus closely matches the burst of spikes in a spiking local interneuron recorded simultaneously. What is the role played by the interposed inhibitory interneurons and the function of these inhibitory regions?

Sharpening the edges of tactile receptive fields. The intersegmental interneuron in Figure 3 is excited by touching hairs on the dorsal tarsus, and, like the one in Figure $2 B$, is inhibited by receptors on the ventral pads (Fig. 3, $A, B$ ). The spiking local interncuron recorded simultancously has the oppositc receptive field. Thus, when the ventral tarsus is touched, the spiking local interneuron spikes faster, and the intersegmental interneuron is hyperpolarized (open arrows, Fig. 3A). If current is injected through a balanced electrode into the local interneuron to make it spike faster, the frequency of IPSPs in the intersegmental interneuron is correspondingly increased (Fig. 3C). Signal averaging reveals that an IPSP follows a spike with a latency of about $1 \mathrm{msec}$ (Fig. $3 D$ ). The boundaries of the excitatory receptive field of this intersegmental interneuron, defined primarily by the array of hairs on the dorsal tarsus that excites it, can therefore be sharpened by lateral inhibition from this presynaptic spiking local interneuron.

Encoding of intensity of contact. Sometimes, a single region of the leg can have both receptors that excite and others that inhibit an intersegmental interneuron. For example, the intersegmental interneuron in Figure 4 is excited when hairs on the dorsoproximal region of the tibia are touched (Fig. $4, A, B$ ). The spiking local interneuron recorded simultaneously is slightly hyperpolarized at each stimulus. If, however, pressure is applied to the cuticle beneath which contains campaniform sensilla, the spiking local interneuron is excited and the excitation of the intersegmental interneuron due to hair movements is overriden by inhibitory inputs caused by cuticular strain (Fig. $4 C$ ). Therefore, if this region of the leg were to encounter an obstacle when the animal was walking, the intersegmental interneuron would be excited if the contact were light, but inhibited if the contact were heavier.

\section{Role of inhibition in the processing of proprioceptive information}

In the same population of intersegmental interneurons are also individuals that respond to movements about one or more joints in the leg, indicating that they receive proprioceptive inputs. Their proprioceptive receptive fields, here defined as the ensemble of joint receptors that affect them, can also contain excitatory and inhibitory regions. One interneuron, for example, is inhibited by an imposed extension of the tibia (Fig. $5 A$ ) and excited by the intrinsic movements of the tarsus (Fig. $5 B$ ). Repeated movements of the tibia evoke a barrage of IPSPs at each imposed extension (Fig. $5 A, i$ ). Hyperpolarizing current passed into the interneuron to suppress its tonic spikes reveals clearly that no EPSPs accompany the flexion of the tibia, so that the slow repolarization of the membrane potential after an extension is due to the cessation of the inhibitory inputs (Fig. 5A, ii). If the tibia is held in an extended position, and movements are then imposed on the second and third tarsal segments, the interneu- 

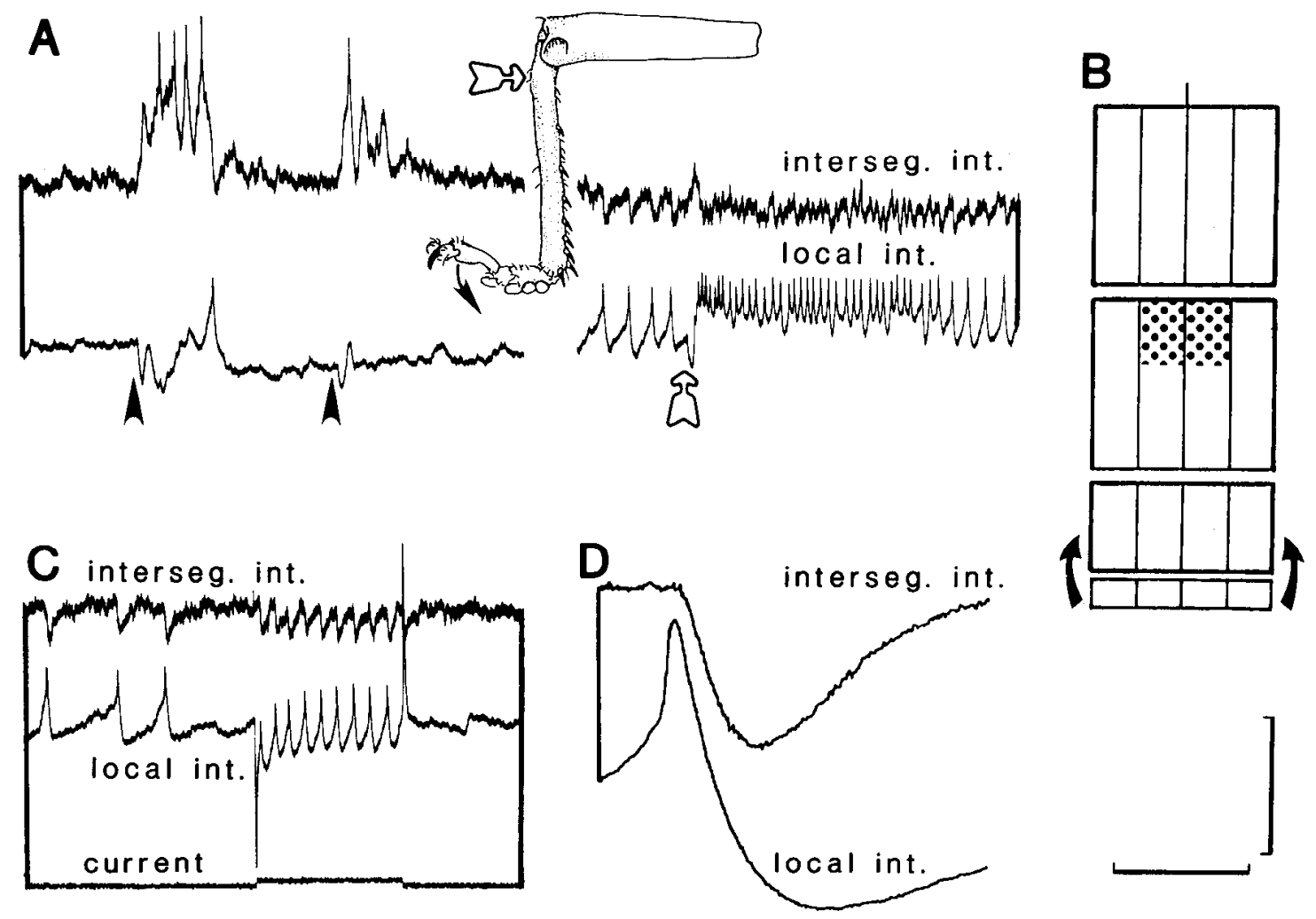

Figure 7. An intersegmental interneuron excited by proprioceptive inputs and inhibited by exteroreceptive stimuli. $A$, The intersegmental interneuron is depolarized by a retraction of the unguis (filled arrowheads), but receives a barrage of high-frequency IPSPs when campaniform sensilla on the dorsal proximal tibia are pressed (open arrow). The local interneuron, by contrast, is hyperpolarized by retraction of the unguis, but strongly excited by pressing on the tibial campaniform sensilla. $B$, Receptive field of the intersegmental interneuron. Stippled area, region of the tibia where exteroreceptive stimulation leads to inhibition; filled arrows, upwards, retraction of the unguis is excitatory. $C$, Spikes in the local interneuron, evoked by intracellular injection of current, evoke 1:1 IPSPs in the intersegmental interneuron. $D$, Signal average of 128 sweeps triggered from spikes in the local interneuron. The latency to the IPSP is about $0.7-0.8 \mathrm{msec}$. Calibration: Horizontal, $0.5 \mathrm{sec}(A, C) ; 17.5 \mathrm{msec}(D)$; vertical, intersegmental, $2.5 \mathrm{mV}$; local, $5 \mathrm{mV}(A, C)$.

ron is initially inhibited, but then excited by each stimulus (arrows, Fig. $5 B$ ). Thus, for this interneuron, excitation and inhibition are each associated with the movement of one joint of the leg.

Other interneurons are excited and inhibited by the movements on only one joint (Fig. $5 \mathrm{C}$ ). If the tibia is flexed, the interneuron is hyperpolarized and its membrane potential remains stable for as long as the femorotibial angle is maintained (Fig. 5C). If the tibia is then extended back to its initial position, the interneuron is phasically depolarized and spikes. A close examination of the variations of membrane potential of this interneuron reveals that the depolarization during extension is due to summating EPSPs, whereas the hyperpolarization during flexion is due to summating IPSPs, in addition to the cessation of the cxcitatory inputs. Thus, excitation and inhibition are each associated here with one direction of movement about this joint.

\section{Mixing of mechanosensory modalities}

Approximately a quarter of the intersegmental interneurons in this population are sensitive to both movement and tactile inputs. Usually, one modality causes excitation and the other inhibition.

The intersegmental interneuron in Figure 6, for example, is excited by hairs on the posterior side of the tibia and tarsus and inhibited by the retraction of the unguis (Fig. 6A). This inhibition is caused by a local interneuron, which is, by contrast, excited by the retraction of the unguis. If steady current is passed into this presynaptic local interneuron, the inhibitory relationship is revealed. An increase in the firing frequency of the local interneuron from 13 to $28.5 \mathrm{~Hz}$ causes a drop in the spike frequency of the intersegmental interneuron from 17 to $8.5 \mathrm{~Hz}$ (Fig. 6B, $i$ ). Conversely, injecting hyperpolarizing current to suppress completely spikes in the local interneuron results in a $65 \%$ increase in the frequency of spikes in the intersegmental interneuron (Fig. 6B, ii). Superimposed sweeps triggered from spikes of the local interneuron show that IPSPs in the intersegmental interneuron follow with a constant latency of about 1 msec (Fig. $6 C$ ). Consequently, each time the posterior tarsus is touched, the intersegmental interneuron is first excited by hair inputs (arrowheads, Fig. 6D), but inhibited immediately by the local interneuron if a movement of the unguis follows (open arrows, Fig. 6D). A spontaneous retraction of the unguis, however, would only involve movement, and would thus inhibit the intersegmental interneuron. This interneuron could therefore provide information about whether the movement was active or passive.

A different intersegmental interneuron is, by contrast, excited by the retraction of the unguis (arrowheads, Fig. $7 A$ ). If, however, pressure is applied to the campaniform sensilla on the proximal tibia, a burst of high-frequency IPSPs is evoked in the intersegmental interneuron (open arrow, Fig. $7 A$ ). This interneuron is therefore excited by proprioceptive and inhibited by 


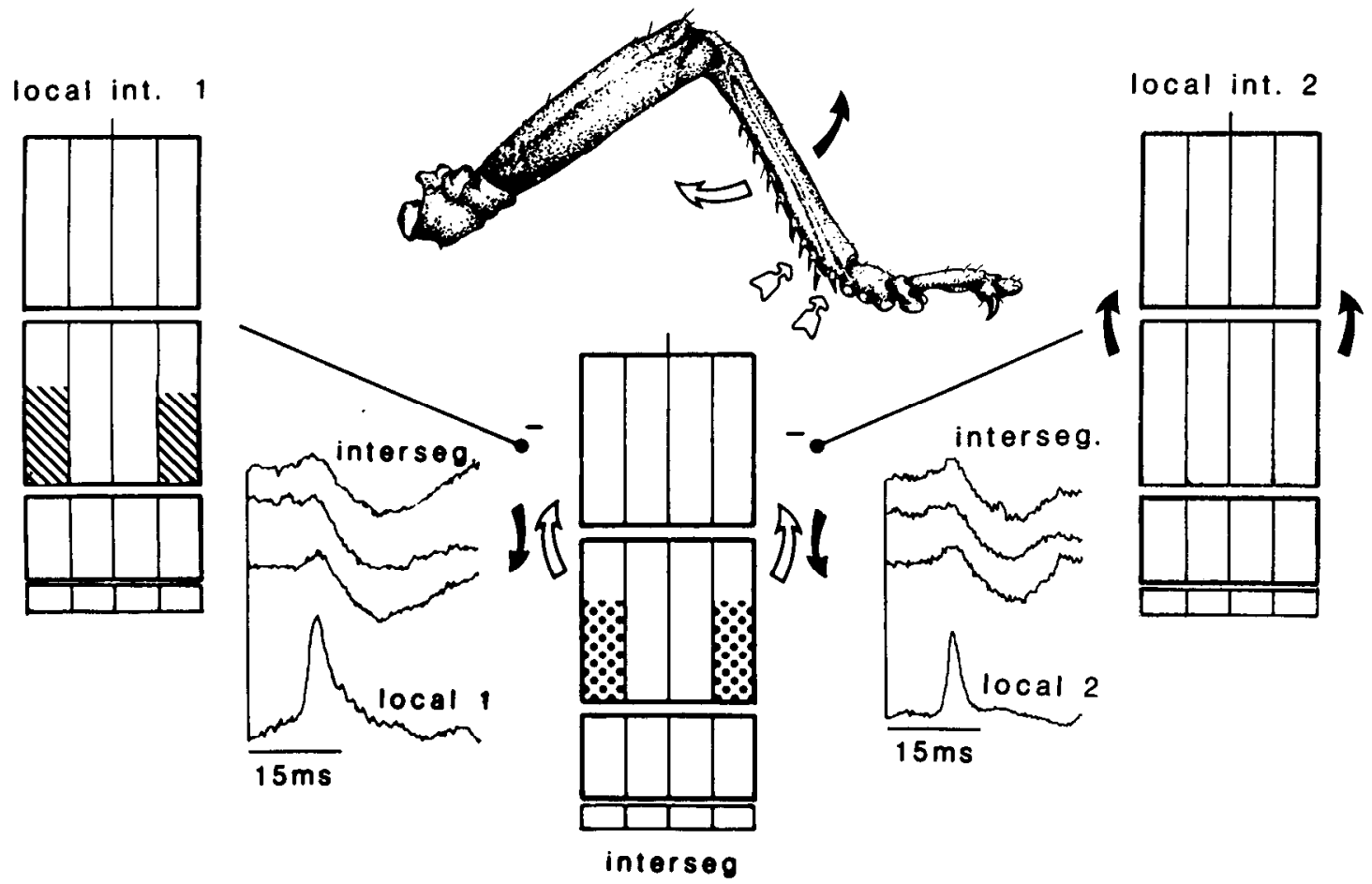

Figure 8. Convergence of inhibitory inputs from 2 local interneurons onto one intersegmental interneuron (center). This intersegmental interneuron is excited by extension of the tibia, and inhibited by flexion of the tibia, or tactile stimulation of the ventral half of the tibia. Local interneuron 1 (left) is excited by tactile inputs on the ventral distal tibia. Its spikes cause an IPSP in the intersegmental interneuron. Local interneuron 2 (right) is excited by the flexion of the tibia. Its spikes also cause an IPSP in the same intersegmental interneuron. Each signal average is of 64 sweeps triggered from spikes in the local interneurons.

exteroreceptive inputs (Fig. 7B). Here, again, the inhibition is caused by a local interneuron that is excited when the tibial campaniform sensilla are pressed (open arrow, Fig. $7 A$ ). Spikes in this local interneuron, occurring spontaneously or evoked electrically, cause an IPSP in the intersegmental interneuron (Fig. 7C) after a latency of just under $1 \mathrm{msec}$ (Fig. 7D).

For these 2 intersegmental interneurons, a voluntary movement of the unguis leads to a simple excitatory or inhibitory response, whereas a retraction occurring in conjunction with tactile inputs, as is likely to take place during walking, leads to a complex summation of inhibitory and excitatory inputs.

\section{Convergence and specificity of the inhihitory inputs}

The inhibition of an intersegmental interneuron can be caused by a single presynaptic local interneuron. For example, when tibial campaniform sensilla are touched, the intersegmental interneuron in Figure 7 receives a barrage of IPSPs that are each derived from spikes in one presynaptic local interneuron (open arrow, Fig. $7 A$ ). If spikes in this local interneuron are suppressed by injection of hyperpolarizing current, the inhibitory region of the intersegmental interneuron is abolished. Hyperpolarizing one presynaptic local interneuron, however, is often not suffcient to suppress the inhibitory inputs to an intersegmental interneuron, indicating that convergence must occur (arrowheads, Fig. $6 B, i i)$.

Certain intersegmental interneurons have several inhibitory regions in their receptive field. One, for example, is inhibited by a flexion of the tibia and by tactile stimulation of the ventrodistal half of the tibia (Fig. 8). It is also excited by the extension of the tibia. By sampling 5 spiking local interneurons while recording from this intersegmental interneuron, we found
2 that inhibit it and thus account for the inhibitory inputs. The first local interneuron is excited by touching hairs on the ventrodistal tibia (local interneuron 1, Fig. 8). The second is excited by flexion of the tibia (local interneuron 2, Fig. 8).

The third local interneuron is excited by flexing (local interneuron 3, Fig. 9A) and inhibited by extending the tibia (Fig. $9 B$ ). Its receptive field is therefore partly the reciprocal of that of the intersegmental interneuron. Nevertheless, injecting current into this local interneuron to make it spike faster has no effect on the intersegmental interneuron (Fig. 9C), nor does signal averaging reveal any connection between them (Fig. 9D). A fourth local interneuron was excited by both the flexion and extension of the tibia, and a fifth by the extension only. Neither had any effect on this intersegmental interneuron (Fig. $9 E$ ).

Even if a local interneuron is excited by the same stimuli that inhibit an intersegmental interneuron, it does not thus necessarily follow, however, that the former inhibits the latter.

\section{Discussion}

What determines the receptive field of an intersegmental interneuron?

The intersegmental interneurons in this mesothoracic population all respond to mechanosensory stimuli from a leg (Laurent, $1986,1987 \mathrm{a})$. The excitatory regions of their receptive fields are determined by the array of proprioceptors and exteroreceptors that make monosynaptic contact with them (Laurent, 1987b, c). The interneurons can also be inhibited by the same mechanosensory stimuli when applied to different regions of the same leg. The inhibition is mediated by onc or scveral (in parallel) interposed spiking local interneurons (Laurent, 1987c, and this paper). The latter interneurons receive direct inputs from ex- 

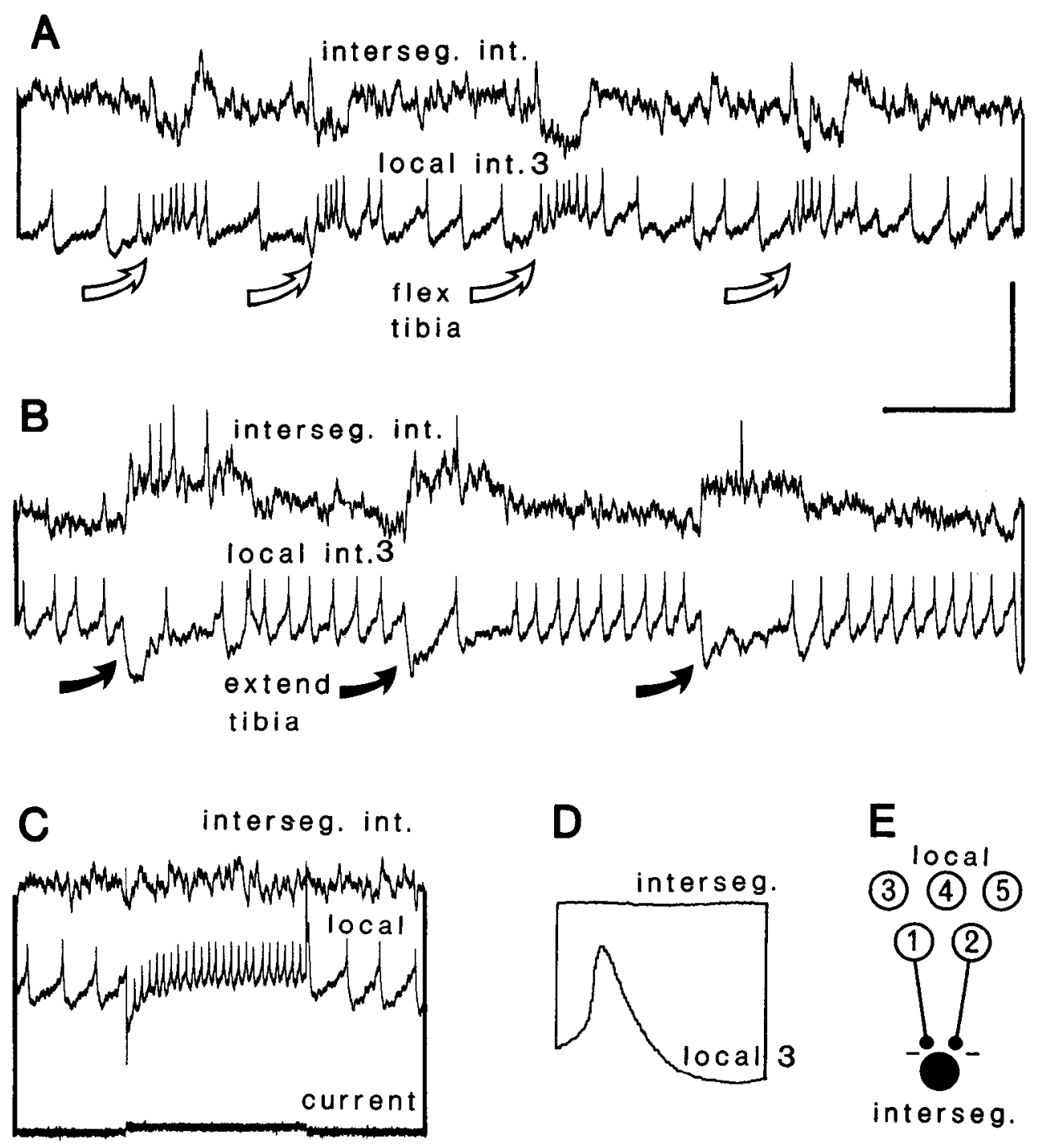

Figure 9. Specificity of the connections. The intersegmental interneuron is the same as in Figure 8. $A$, When the tibia is flexed, local interneuron 3 is depolarized and spikes faster, whereas the intersegmental interneuron is hyperpolarized by a barrage of IPSPs. $B$, When the tibia is extended, the opposite occurs: the intersegmental interneuron is excited, whereas the local one is inhibited. $C$, If current is injected in local interneuron 3 to increase its spike frequency, however, no effect is seen in the intersegmental interneuron. $D$, Signal averaging 128 sweeps triggered from the spike of local interneuron 3 reveals no connection. $E$, This intersegmental interneuron receives IPSPs from 2 (Fig. 8) of 5 sampled local interneurons that had an excitatory region in their receptive field that overlapped with the inhibitory receptive field of the intersegmental interneuron. Calibration: Horizontal, $0.5 \sec (A-C) ; 14 \mathrm{msec}(D)$; vertical, $5 \mathrm{mV}(A-C)$. teroreceptors (Siegler and Burrows, 1983; Burrows and Pflüger, 1986) and proprioceptors (Burrows, 1987a; Pflüger and Burrows, 1987), so that the inhibitory regions in the receptive field of an intersegmental interneuron are determined by the array of receptors that synapse onto the spiking local interneuron(s) presynaptic to it. In all examples where a connection was found, the entire excitatory region of the receptive field of a spiking local interneuron was never larger (but sometimes smaller) than the inhibitory region of the receptive field of the postsynaptic intersegmental interneuron. This finding is summarized in Figure $10 \mathrm{~A}$ for an intersegmental interneuron with a tactile receptive field comprising one excitatory and two inhibitory regions. It will receive direct inputs from an array of afferents on the leg (3, Fig. 10A) and inhibitory inputs from 2 spiking local interneurons interposed between it and the other 2 arrays of afferents ( 1 and 2, Fig. 10A). Similarly, an interneuron sensitive to movement about one joint will be excited by afferents coding for one direction and inhibited by a local interneuron that is itself excited by the afferents coding for the opposite direction (Fig. 10B).

Intersegmental interneurons with more complex receptive fields (e.g., with mixed mechanosensory modalities) will still use the same principles of connectivity. The receptive fields of all 26 intersegmental interneurons in Figure 11 can be explained by these patterns of connections and the known properties of the spiking local interneurons (Burrows, 1985; Burrows and Siegler, 1985).

Beside the monosynaptic excitation and disynaptic inhibition of the intersegmental interneurons, a process of disinhibition, probably involving 2 interposed local interneurons in series, also refines their receptive fields. A stimulus that excites an intersegmental interneuron often inhibits the presynaptic local interneuron (e.g., Figs. $3 A, 7 A$ ). This pathway provides a supplementary source of excitation because it decreascs or suppresses the inhibition mediated by the spiking local interneuron.

\section{Specificity of the inhibitory connections}

Not all spiking local interneurons excited by the same stimulus inhibit a particular intersegmental interneuron. For example, an intersegmental interneuron inhibited by flexion of the tibia receives IPSPs from only one of 3 sampled local interneurons that are excited by this movement. For one of the 2 interneurons that do not connect, the reason is clear: it is also excited by extension of the tibia, so that it would mediate an unwanted inhibition to this movement as well. For the second interneuron (Fig. 9), the reason is less obvious. It is, however, becoming more and more evident that any given mechanical stimulation 
A

Afferents Local ints. Interseg. ints.

Figure 10. Established patterns of connections between sensory afferents and local and intersegmental interneurons. $A$, The excitatory regions of the tactile receptive field of an intersegmental interneuron are caused by direct connections with the afferents (3) (Laurent, $1987 \mathrm{c}$ ). The inhibitory regions are due to inhibitory inputs from one (or several, in parallel) interposed spiking local interneuron $(1,2)$ (Laurent, 1987c, and this paper). $B$, Pathways for proprioceptive inputs (see also Laurent, 1987b). The stimuli that excite an intersegmental interneuron often inhibit the local interneuron responsible for its lateral inhibition. The connections between the local and intersegmental interneurons being unidirectional, this inhibition thus is possibly due to inhibitory interactions between the spiking local interneurons themselves (dashed lines). $e, f$, Local interneurons respectively excited by extension and flexion of a joint.

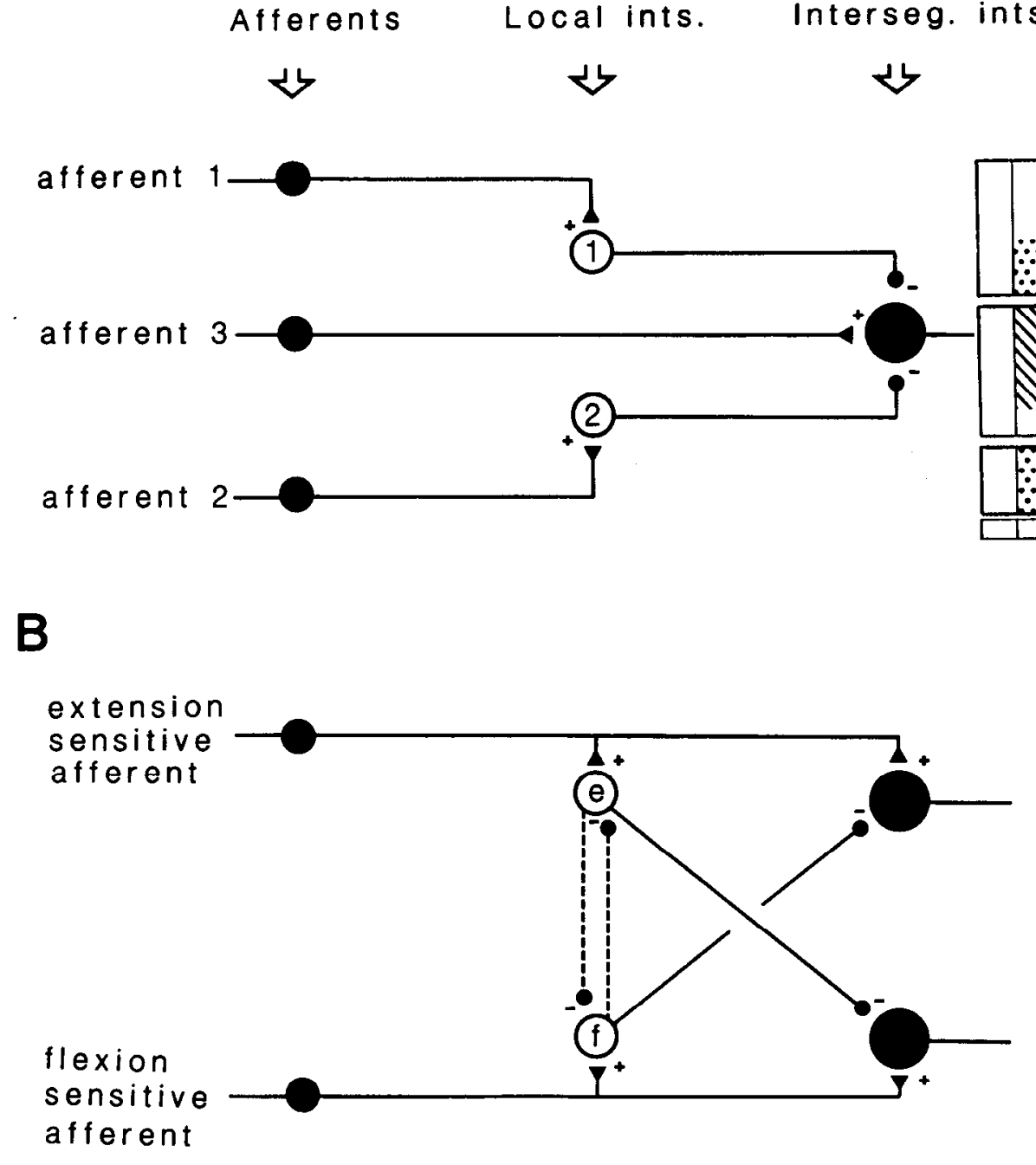

(movement, touch, pressure, etc.) is encoded in the periphery by a very large number of sense organs, each of which is sensitive to only a few parameters of the stimulus. Tibial movements alone, for example, can excite chordotonal afferents (Burns, 1974), a strand rcccptor (Bräunig, 1985), multipolar "joint reccptors," (Coillot and Boistel, 1968), hairs at the femorotibial joint, and campaniform sensilla monitoring strain of the cuticle. The specificity of coding by these afferents is retained in the connections they make with spiking local interneurons. Chordotonal afferents connect with certain local interneurons (Burrows, 1987a), the strand receptor with one or 2 of these (Pflüger and Burrows, 1987). Exteroreceptors excite other local interneurons, among which some also receive proprioceptive inputs (Siegler and Burrows, 1983). The definition of a stimulus as "tibial extension," or "touch of the tarsus," is thus insufficient to describe its precise characteristics. It follows that during such an imposed stimulus, several local interneurons will be excited in parallel. The specificity of the connections between local and intersegmental interneurons therefore reflects that of the connections between the afferents and the local interneurons. Thus the inhibition of an intersegmental interneuron is not only determined by the type of stimulation (c.g., movement or touch), but by the nature of the receptor(s) excited by the stimulation.
During the processing of mechanosensory information, the specificity of the peripheral signals thus seems to be preserved throughout the successive stages of integration by first- and second-order sensory interneurons.

\section{Role of the inhibition}

If an intersegmental interneuron has excitatory and inhibitory regions in its tactile receptive field, and if these 2 regions are contiguous, the inhibition can be seen as a means to sharpen the edges of the excitatory regions and thereby to increase the contrast. The interneuron in Figure 3, for example, will be excited best by tactile inputs on the dorsal surface of the tarsus, and inhibited when the foot is in contact with the ground. This means that its response will be greatest if the fooi meets an obstacle during the swing phase of a step cycle, when it is off the ground.

The intensity of tactile stimuli encoded in the periphery by different afferents is enhanced by inhibition. The summed inputs are transmitted to the intersegmental interneurons in the form of excitation (for a light contact-caused movement of hairs) and inhibition (for movement or strain of the cuticle, causing deformation of campaniform sensilla).

During movement, inhibition provides temporal accuracy. 

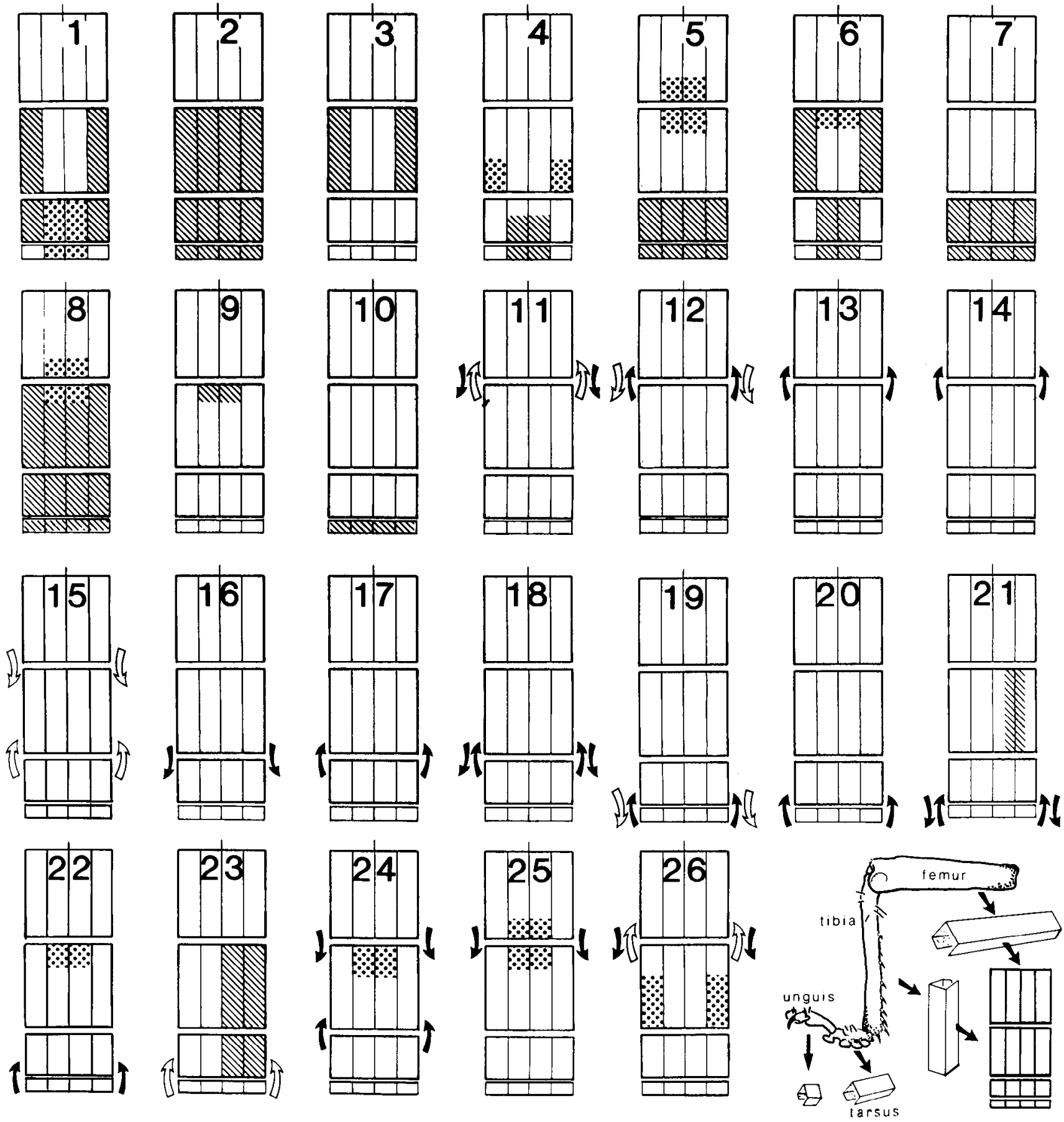

Figure 11. The receptive fields of 26 intersegmental interneurons in this mesothoracic population (Laurent, 1987a), sensitive to exteroreceptive stimuli only $(1-10)$, proprioceptive stimuli only $(11-20)$, or both $(21-26)$. The difference between interneurons 13 and 14 is that one is excited by voluntary movement only, whereas the second is excited by voluntary as well as imposed flexion. Stippled areas, tactile inhibitory regions; hatched areas, excitatory. Filled arrows, excitatory; open arrows, inhibitory.

Interneuron 11 in Figure 11 is excited when the tibia is extended. The inhibition provided by an interposed spiking local interneuron ensures that its membrane is repolarized during flexion and that it spikes only during extension (see, for example, Fig. $5 C$ ). During extensions and flexions of the tibia at $10 \mathrm{~Hz}$, the interneuron will spike precisely in phase with the movement because the inhibition immediately shuts off the spikes during flexion. By contrast, a neuron whose responses to movement about one joint are controlled only by excitation or only by inhibition cannot follow rapidly changing stimuli with accuracy, since its excitation or inhibition generally outlasts the stimuli (see Fig. $5 A, i i, B$ ).

\section{Complexity of the receptive fields of the intersegmental interneurons}

Some interneurons have simple receptive fields and are, for example, excited only by pressure on the proximal tibia, or by flexion of the tibia. Others have complex combinations of 
excitatory and inhibitory zones that involve different mechanosensory modalities (e.g., interneurons 21 and 26, Fig. 11). The complexity of these sensory receptive fields can, however, be explained if they are envisaged during walking. At the beginning of the stance phase, the leg reaches the ground and grasps it to pull the body forward, so that there is strain on the tibia (Zill and Moran, 1981) and a retraction of the unguis. Interneuron 22 (Fig. 11) would be excited by the retraction of the unguis, but this would be curtailed by the inhibition resulting from strain in the tibia. As a result, spikes are prevented. This interneuron is therefore likely to be excited most when the leg does not support the body weight at the end of the stance phase. Similarly, interneuron 25 (Fig. 11) is excited by extension of the tibia, but inhibited by the hairs on the knee joint that are bent when the leg is fully extended. This interneuron will therefore respond best at femorotibial angles below $130^{\circ}-150^{\circ}$, the range displayed during walking (Burns, 1974).

The complex representation of primary sensory signals from the leg by certain intersegmental interneurons requires the existence of other intersegmental interneurons with restricted receptive fields (e.g., 9 and 14, Fig. 11) to preserve cerlain basic signals (for example, the nature or location of the stimulus). A burst of spikes in interneuron 8 (Fig. 11), for example, signals that the tibia, tarsus, and/or unguis have hit an obstacle, but gives only diffuse spatial information. Interneuron 10 (Fig. 11) will, by contrast, indicate that the contact occurred on the unguis. It follows from these observations that a certain degree of convergence of the outputs from these intersegmental interneurons should be expected on their metathoracic targets.

\section{How widespread is this principle of organization?}

In cricket and cockroaches, a population of "giant interneurons" in the terminal ganglion responds to mechanoreceptive inputs delivered to the cerci (Edwards and Palka, 1974). These interneurons respond to airborne vibrations with great directional sensitivity, owing to converging excitatory and inhibitory inputs in their receptive fields (Jacobs et al., 1986). Excitation appears to be provided directly by the hair afferents (Callec et al., 1971; Bacon and Murphey, 1984; Blagburn et al., 1984), whereas inhibition is both pre- and postsynaptic (Levine and Murphey, 1980). For instance, stimulation of certain posterior "L-hairs" on either cercus results in a hyperpolarization of the pair of "10-3" interneurons (Jacobs et al., 1986). Although no local inhibitory interneurons have yet been described in the terminal ganglia of orthoptera, the existence of such neurons should be expected.

In the crayfish, similar ascending mechanosensory interneurons respond with directional sensitivity to water displacements (Wiese et al., 1976). The excitatory regions in their receptive fields are due to monosynaptic inputs from hairs on the telson (Wiese et al., 1976), and their inhibitory regions to lateral inhibition from interposed nonspiking local interneurons (Reichcrt ct al., 1983).

It can be predicted that, in invertebrates at least, local or unisegmental interneurons serve not only to mediate local reflexes (Burrows and Siegler, 1982) and to shape motor outputs (Burrows, 1987b), but, in addition, to sharpen the receptive fields of intersegmental interneurons. What is now needed is to understand how the specific signals transmitted by intersegmental interneurons interact with distant local centers. The population of intersegmental interneurons studied here presents a rare opportunity in that they project to a ganglion where the local circuits controlling the movements of a leg are known in great detail.

\section{References}

Bacon, J. P., and J. S. Altman (1977) A silver intensification method for cobalt-filled neurones in wholemount preparations. Brain Res. 138: 359-363.

Bacon, J. P., and R. K. Murphey (1984) Receptive fields of cricket giant interneurons are determined by their dendritic structure. J. Physiol. (Lond.) 352: 601-623.

Blagburn, J. M., D. J. Beadle, and D. B. Satelle (1984) Synapses between an identified giant interneurone and a filiform hair sensory neurone in the terminal ganglion of first instar cockroaches (Periplaneta americana L.). J. Exp. Biol. 113: 477-481.

Bräunig, P. (1985) Strand receptors associated with the femoral chordotonal organs of locust legs. J. Exp. Biol. 116: 331-341.

Brogan, R. T., and R. M. Pitman (1981) Axonal regeneration in an identified insect motoneurone. J. Physiol. (Lond.) 319: 34P-35P.

Burns, M. D. (1974) Structure and physiology of the locust femoral chordotonal organ. J. Insect Physiol. 20: 1319-1339.

Burrows, M. (1985) The processing of mechanosensory information by spiking local interneurones in the locust. J. Neurophysiol. 54:463478.

Burrows, M. (1987a) Parallel processing of proprioceptive signals by spiking local interneurons and motor neurons in the locust. J. Neurosci. 7: 1064-1080.

Burrows, M. (1987b) Inhibitory interactions between spiking and nonspiking local interneurons in the locust. J. Neurosci. (in press).

Burrows, M., and H. J. Pflüger (1986) Processing by local interneurons of mechanosensory signals involved in a leg reflex of the locust. J. Neurosci. 6: 2764-2777.

Burrows, M., and M. V. S. Siegler (1982) Spiking local interneurons mediate local reflexes. Science 217: 650-652.

Burrows, M., and M. V.S. Siegler (1985) The organization of receptive fields of spiking local interneurons in the locust with inputs from hair afferents. J. Neurophysiol. 53: 1147-1157.

Burrows, M., and B. L. Watkins (1986) Spiking local interneurones in the mesothoracic ganglion of the locust: Homologies with metathoracic interneurones. J. Comp. Neurol. 245: 29-40.

Callec, J. J., J. C. Guillet, Y. Pichon, and J. Boistel (1971) Further studies on synaptic transmission in insects. II. Relations between sensory information and its synaptic integration at the level of a single giant axon in the cockroach. J. Exp. Biol. 55: 123-149.

Cervero, F. (1986) Dorsal horn neurones and their sensory inputs. In Spinal Afferent Processing, T. L. Yaksh, ed., pp. 197-216, Plenum, New York.

Coillot, J. P., and J. Boistel (1968) Localisation et description des recepteurs a l'etirement au niveau de l'articulation tibio-femorale de la patte sauteuse du criquet Schistocerca gregaria. J. Insect Physiol. 14: 1661-1667.

Edwards, J. S., and J. Palka (1974) The ccrci and abdominal giant fibres of the house cricket Acheta domesticus. I. Anatomy and physiology of normal adults. Proc. R. Soc. Lond. [Biol.] 185: 83-103.

Jacobs, G. A., J. P. Miller, and R. K. Murphey (1986) Integrative mechanisms controlling directional sensitivity of an identified sensory interneuron. J. Neurosci. 6: 2298-2311.

Laurent, G. (1986) Thoracic intersegmental interneurones in the locust with mechanoreceptive inputs from a leg. J. Comp. Physiol. A 159: 171-186.

Laurent, G. (1987a) The morphology of a population of thoracic intersegmental interneurones in the locust. J. Comp. Neurol. 256:412429.

Laurent, G. (1987b) Parallel effects of joint receptors on motor neurones and intersegmental interneurones in the locust. J. Comp. Physiol. $\Lambda$ 160: 341-353.

Laurent, G. (1987c) Local circuits underlying excitation and inhibition of intersegmental interneurones in the locust. J. Comp. Physiol. (in press).

Levine, R. B., and R. K. Murphey (1980) Pre- and postsynaptic inhibition of identified giant interneurones in the cricket (Acheta domesticus). J. Comp. Physiol. A 135: 269-282.

Pflüger, H. J., and M. Burrows (1987) A strand receptor with a central 
cell body synapses upon spiking local interneurones in the locust. J. Comp. Physiol. A 160: 295-304.

Pitman, R. M., C. D. Tweedle, and M. J. Cohen (1972) Branching of central neurones: Intracellular cobalt injection for light and electron microscopy. Science 176: 412-414.

Price, D. D. (1986) The question of how the dorsal horn encodes sensory information. In Spinal Afferent Processing, T. L. Yaksh, ed., pp. 445-466, Plenum, New York.

Reichert, H., M. R. Plummer, and J. J. Wine (1983) Identified nonspiking local interneurons mediate nonrecurrent lateral inhibition of crayfish mechanosensory interneurons. J. Comp. Physiol. A 151:261276.

Siegler, M. V. S., and M. Burrows (1983) Spiking local interneurons as primary integrators of mechanosensory information in the locust. J. Neurophysiol. 50: 1281-1295.

Siegler, M. V. S., and M. Burrows (1986) Receptive fields of motor neurons underlying local tactile reflexes in the locust. J. Neurosci. 6 : 567-573.

Watson, A. H. D., and M. Burrows (1985) The distribution of synapses on the two fields of neurites of spiking local interneurones in the locust. J. Comp. Neurol. 240: 219-232.

Wiese, K., R. L. Calabrese, and D. Kennedy (1976) Integration of directional mechanosensory inputs by crayfish interneurones. J. Neurophysiol. 39: 834-843.

Wong, R. K. S., and K. J. Pearson (1976) Properties of the trochanteral hairplate and its function in the control of walking in the cockroach. J. Exp. Biol. 64: 233-249.

Zill, S. N., and D. T. Moran (1981) The exoskeleton and insect proprioception. III: Activity of tibial campaniform sensilla during walking in the American cockroach, Periplaneta americana. J. Exp. Biol. 94: $57-75$. 